\title{
Surgical management of left ventricular posterior wall plication for cardiac shock with ischemic mitral regurgitation
}

\author{
Kosuke Nakamae ${ }^{1}$, Takashi Oshitomi ${ }^{2}$, and Hideyuki Uesugi ${ }^{2}$ \\ ${ }^{1}$ Saiseikai Kumamoto Hospital \\ ${ }^{2}$ Saiseikai Kumamoto Byoin
}

January 22, 2021

\begin{abstract}
Ischemic mitral regurgitation (IMR) is a common complication, which is accompanied by myocardial infarction, causing heart failure and leading to poor prognosis. Although several surgical techniques have been reported, certain surgical methods have not been established for treating IMR. We report a successful case of left ventricular posterior wall plication through a left atriotomy over the mitral valve for IMR in a patient who experienced cardiac shock and could not be weaned off mechanical support. Posterior wall plication changed the left ventricle from a spherical to an oval shape, restored the position of papillary muscles and posterior wall, improved leaflet tethering, and prevented further remodeling of the left ventricle. This method may be useful for treating IMR and improve patients' prognosis.
\end{abstract}

\section{Introduction}

Ischemic mitral regurgitation (IMR), a well-known complication of myocardial infarction (MI), is subcategorized as a secondary mitral regurgitation (MR) and is a well-known cause of heart failure, leading to a poor prognosis. ${ }^{1}$ Surgical techniques like undersized annuloplasty, papillary muscle relocation or approximation, and chordal cutting have been reported previously, but certain surgical methods have not been established for treating IMR. We report a successful case of left ventricular posterior wall plication (LVPWP) for a patient in cardiac shock with severe IMR shock that could not be weaned from mechanical support.

\section{Case}

A 73-year-old man with a history of diabetes mellitus was referred to our hospital for treating coronary artery disease. Coronary artery angiography showed severe diffuse stenosis of the left anterior descending artery (LAD) and old MI of the left circumflex artery ( $\mathrm{LCx}$ ), with a collateral flow from the LAD to the LCx (Figure 1a, b). We performed percutaneous coronary intervention (PCI) for the LAD and LCx that led to coronary LAD perforation (Figure 1c). To repair this, we implanted a covered stent in the LAD and jailed the septal and two large diagonal branches (D1, D2) (Figure 1d). The patient experienced cardiac shock, was intubated, and the Impella CP (Abiomed, Danvers, Massachusetts, USA) was inserted. One week later, the patient was weaned off the Impella $\mathrm{CP}$ and it was removed, with moderate MR being present; however, 2 days later, it was re-inserted because of pulmonary congestion and low output syndrome due to the worsening of MR. Echocardiography under Impella CP support showed a low ejection fraction (EF) of $31 \%$, severe MR with tethering, and global hypokinesis, compared to an EF of $57 \%$, trivial MR, and only basal infero-posterior mild hypokinesis before PCI (Figure 2). Mechanical support was difficult to remove due to severe IMR and, therefore our heart team considered a surgery.

Operative procedures 
A cardiopulmonary bypass $(\mathrm{CPB})$ was initiated with the ascending aorta and superior and inferior vena cava under general anesthesia. The hematoma at the site of coronary perforation and thinness at D1, D2, and the apex indicated a broad MI. We performed coronary artery bypass grafting on five vessels with the left internal thoracic artery and saphenous vein, mitral annuloplasty with $32 \mathrm{~mm}$ of CG Future (Medtronic, Dublin, Ireland), and LVPWP through a left atriotomy over the mitral valve (MV). The operative, CPB, and cross-clamp times were $544 \mathrm{~min}, 297 \mathrm{~min}$, and $194 \mathrm{~min}$, respectively. Surgical technique: LVPWP (Figure $3 \mathrm{a})$

We approached the MV through left atriotomy and from over the MV. During LVPWP, we sutured the trabeculae carneae with five horizontal mattress stitches (4-0 polyvinylidene fluoride sutures with small felt strips) to lightly plicate only the endocardial side, not in the deep layer, from the apical side of the bilateral papillary muscle to under the MV.

The hemodynamics stabilized gradually, and the patient was weaned from the Impella CP after 10 postoperative days (POD), extubated after 16 POD, discharged from the intensive care unit after 20 POD, and transferred to another hospital for further rehabilitation after 51 POD. Echocardiography performed 1 month and 1 year postoperatively showed MR was controlled to a trivial level despite a low EF and global hypokinesis (Figure $3 \mathrm{~b}$ ), and there was no recurrence of heart failure at 1 year postoperatively.

\section{Discussion}

The cause of IMR is the remodeling of the left ventricle (LV), a change in the LV from an oval to a spherical shape, and the subsequent displacement of the papillary muscles, causing leaflet tethering and coaptation mismatch without structural valve lesions. ${ }^{2}$ Therefore, additional subvalvular or ventricular procedures are needed to treat IMR.

Liel-Cohen et al. ${ }^{3}$ reported that the plication of the sheep's LV infero-posterior wall in an MI site shortens the distance between both the papillary muscle and annular and bilateral papillary muscles, leading to reduced tethering. Matsui et al. ${ }^{4}$ reported integrated overlapping ventriculoplasty combined with papillary muscle plication using 3 stitches with small felt strips to plicate the bilateral papillary muscle. According to these reports, plication of both the LV infarction site and papillary muscle is a useful method for IMR surgery. However, these methods require left ventriculotomy. The Surgical Treatment for Ischemic Heart Failure trial ${ }^{5}$ showed that LV plasty with left ventriculotomy for IMR did not improve patients' prognosis. Moreover, LV plasty with left ventriculotomy poses a risk of postoperative bleeding, dangerous arrhythmia, and cutting the normal myocardium, causing cardiac dysfunction.

We have performed LVPWP through a left atriotomy over the MV without left ventriculotomy for many cases with IMR, and the long-term outcomes have been relatively favorable. The plication site is ususally determined through preoperative echocardiography, which helps visualize the site of wall thinning and hypokinesis. The trabeculae carneae are then sutured with a horizontal mattress to lightly plicate only the endocardial side. We think performing LVPWP changes the LV from a spherical to an oval shape, restores the position of papillary muscles, improves leaflet tethering, and prevents further LV remodeling. Additionally, this method is advantageous in terms of anatomical limitation and postoperative cardiac expandability compared to LV plasty with left ventriculotomy.

In the present case, our approach improved leaflet tethering and prevented further LV remodeling, enabling the patient to be off mechanical support and a respiratory ventilator. MR was controlled to a trivial level and no heart failure symptoms recurred.

\section{Conclusion}

LVPWP may be a valid surgical strategy for IMR as it improves leaflet tethering, prevents further LV remodeling, and controls MR. Further follow-ups are needed to evaluate the impact of LVPWP on IMR.

\section{Author Contributions}


Kosuke Nakamae: drafting article and doctor-in-chief, Takashi Oshitomi: primary surgeon, Hideyuki Uesugi: approval of article and supervisor

\section{References}

1. Trichon BH, Felker GM, Shaw LK, Cabell CH, O'Connor CM. Relation of frequency and severity of mitral regurgitation to survival among patients with left ventricular systolic dysfunction and heart failure. Am J Cardiol 2003;91:538-543.

2. Otsuji Y, Handschumacher MD, Schwammenthal E, et al. Insights from three-dimensional echocardiography into the mechanism of functional mitral regurgitation: direct in vivo demonstration of altered leaflet tethering geometry. Circulation 1997;96:1999-2008.

3. Liel-Cohen N, Guerrero JL, Otsuji Y, et al. Design of a new surgical approach for ventricular remodeling to relieve ischemic mitral regurgitation: insights from 3-dimensional echocardiography. Circulation 2000;101:2756-2763.

4. Matsui Y, Fukada Y, Naito Y, Sasaki S. Integrated overlapping ventriculoplasty combined with papillary muscle plication for severely dilated heart failure. J Thorac Cardiovasc Surg 2004;127:1221-1223.

5. Jones RH, Velazquez EJ, Michler RE, et al. Coronary bypass surgery with or without surgical ventricular reconstruction. N Engl J Med 2009;360:1705-1117.

\section{Figure Legends}

\section{Figure 1}

Coronary artery angiography showed severe diffuse stenosis of the LAD and old MI of the LCx with a collateral flow from the LAD to the LCx $(a, b)$. During the PCI, coronary perforation of the LAD occurred (c), and the covered stent was implanted, but it jailed the septal and two large diagonal branches (d).

White arrow: diagonal branch, blue arrow: septal branch, purple arrow: collateral flow

Figure 2

Echocardiography after PCI showing global hypokinesis and severe MR due to tethering.

\section{Figure 3}

a: A schematic illustration showing the technique of LVPWP.

Red arrow: horizontal mattress suture with small felt strip, green arrow: anterior papillary muscle, yellow arrow: posteromedial papillary muscle, AC: anterolateral commissure, PC: posteromedial commissure, AML: anterolateral mitral leaflet, PML: posterior mitral leaflet, LV: left ventricular, LA: left atrium

b: Postoperative echocardiography 1 month postoperatively showing that MR was controlled to a trivial level. 


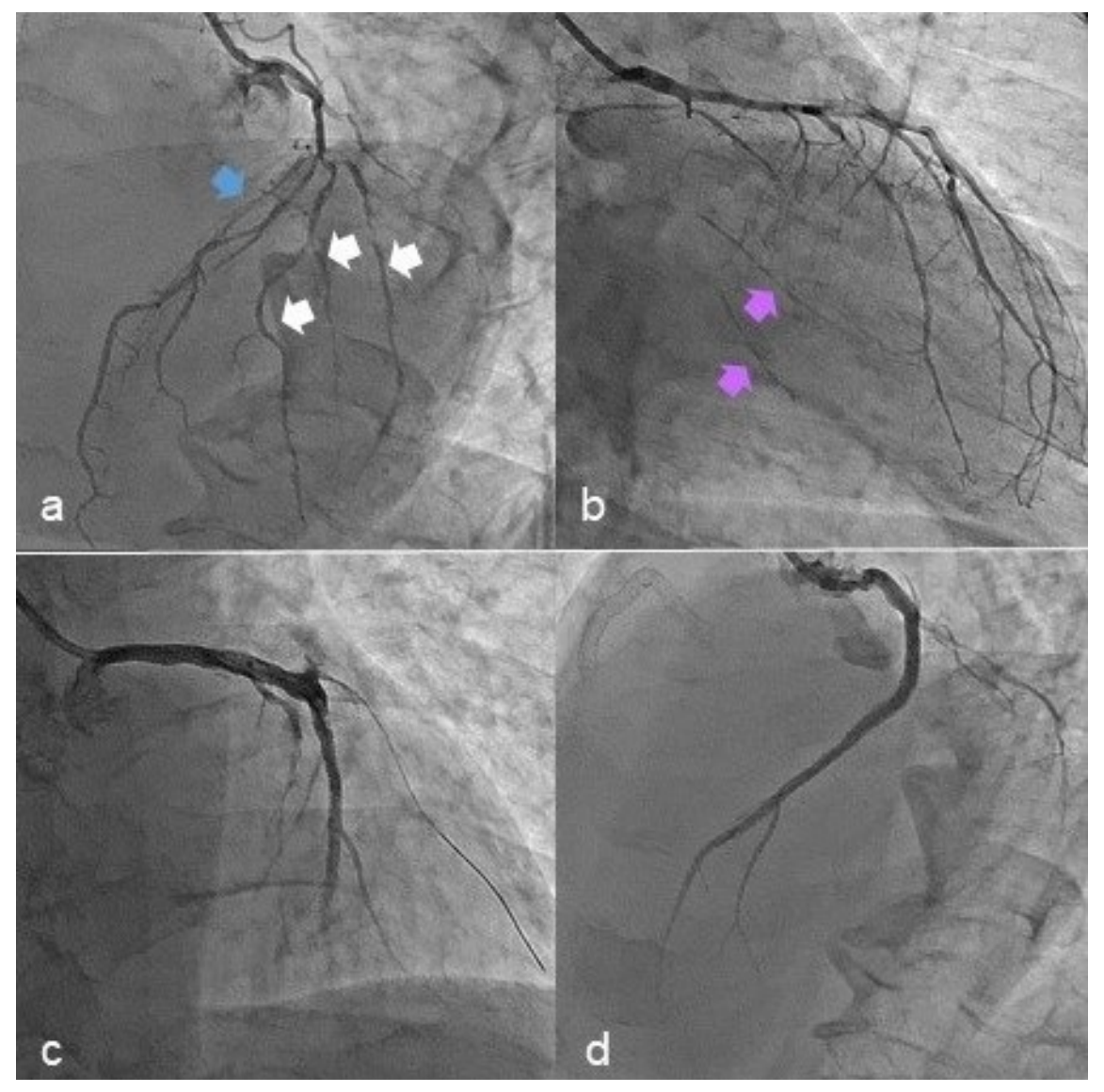



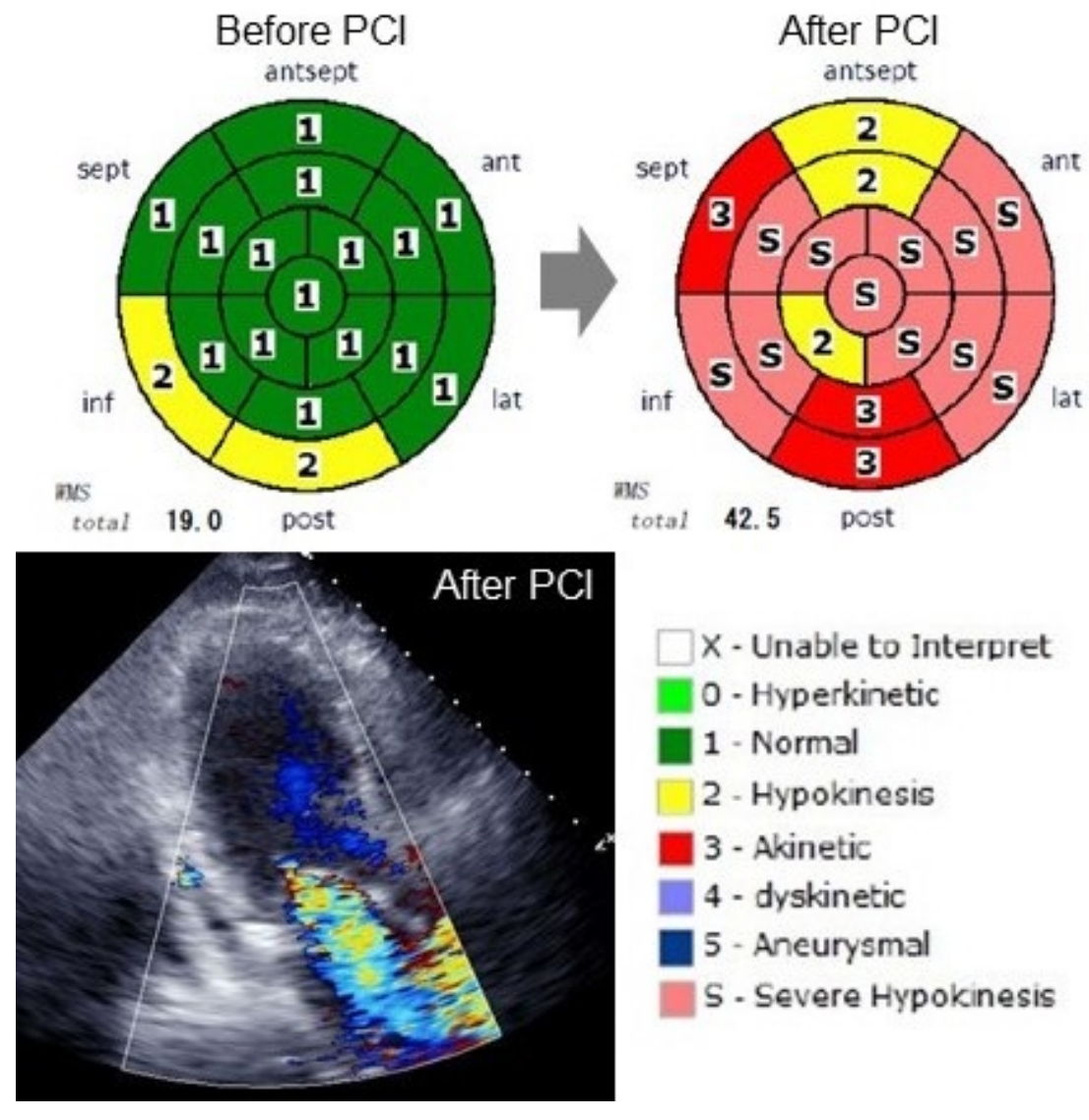

$\square$ X - Unable to Interpret

0 - Hyperkinetic

1 - Normal

2 - Hypokinesis

3 - Akinetic

4 - dyskinetic

5 - Aneurysmal

S. Severe Hypokinesis
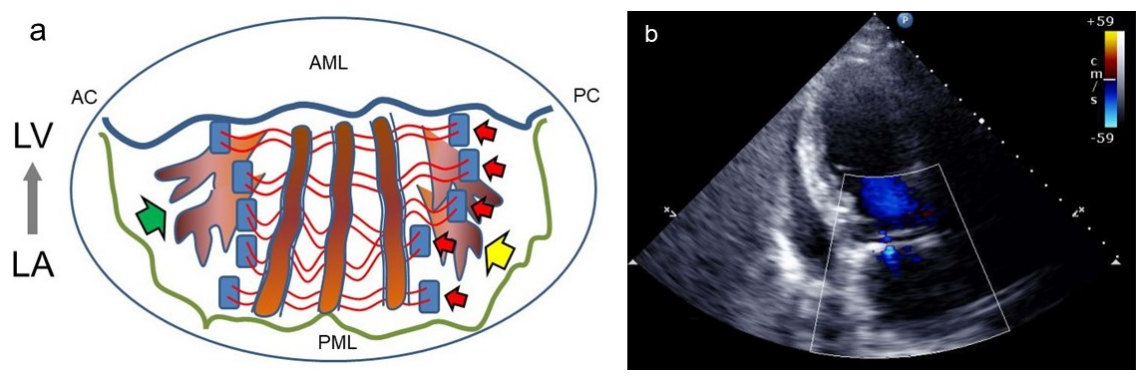